Revista Eletrônica de Farmácia

Eletronic Journal of Pharmacy

ISSN 1808-0804

doi 10.5216/ref.v17.51302

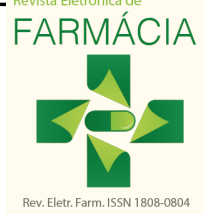

Artigo Original

\title{
Níveis de ansiedade em alunos concluintes de cursos de saúde
}

\section{Anxiety levels in graduating health courses' students}

\section{Niveles de ansiedad en alumnos que concluyen cursos de salud}

\author{
SANTOS, Rômulo Moreira dos ${ }^{1}$. SIMÕES, Monica Oliveira da Silva ${ }^{2 *}$ \\ ${ }^{1}$ Centro Universitário Unifacisa - UNIFACISA . ${ }^{2}$ Universidade Estadual da Paraíba - UEPB \\ *romulomoreirasantos@hotmail.com
}

Resumo. Introdução: A ansiedade é uma condição caracterizada por momentos de tensão ou desconforto, causados por sentimentos de medo e apreensão, mesmo sem motivos aparentes que, de maneira geral, diminuem a qualidade de vida dos portadores. Os estudantes universitários são um grupo susceptível a estados ansiosos. Objetivos: Determinar a prevalência de alunos ansiosos dentre os concluintes de cursos de saúde de faculdades públicas e privadas. Métodos: Realizou-se um estudo transversal em Campina Grande-PB, nos meses de outubro de 2013 a dezembro de 2014, após aprovação por Comitê de Ética, aplicando-se o Beck Anxiety Inventory, validado para o português, e um questionário semiestruturado. Os dados obtidos foram tratados no Statistical Package for The Social Sciences na versão 18, empregando intervalo de confiança de $95 \%$ nas análises calculadas. Resultados: A partir das respostas, foi encontrada relação estatística significante entre os universitários ansiosos e as variáveis horas de sono por dia, qualidade do sono, Instituição de Ensino Superior, insônia, fadiga e medo de ficar só. $\mathrm{Na}$ análise de variância, o curso Farmácia foi o de maior média dos scores do inventário. Conclusão: Concluiu-se que a alta prevalência de universitários ansiosos indica a necessidade de políticas de atenção à saúde mental que atendam esse segmento populacional, melhorando a qualidade de vida nas universidades.

Palavras-chave: Saúde mental. Universitários. Transtorno de ansiedade.

Abstract. Introduction: Anxiety is a disorder characterized by moments of tension or discomfort due feelings of fear and apprehension. Even without apparent reasons that, in general, this decreases the quality of life. Objective: Whereas university students are a group susceptible to anxious states, the aim was to inquire the prevalence of anxiety among graduating university students from public and private health courses. Methodology: A cross-sectional study was carried out in Campina Grande-PB, from October 2013 to December 2014 through Beck Anxiety Inventory Data were analyzed in Statistical Package for The Social Sciences with a 95\% confidence interval. Results: A statistically significant relationship was found between the anxiety and the variables sleep hours per day, sleep quality, educational institution, insomnia, fatigue and fear of being alone. The Pharmacy course was the largest average of the inventory scores. Conclusion: Thus, the prevalence of anxiety among university students indicates the need for mental health care policies in this population segment, improving the quality of life during graduation. Key-words: Mental health. College students. Anxiety disorder.

Resumen. Introducción: La ansiedad es una condición caracterizada por momentos de tensión o malestar, causados por sentimientos de miedo y aprehensión, incluso sin motivos aparentes que, de manera general, disminuyen la calidad de vida de los portadores. Objetivo: De esta forma, el objetivo fue determinar la prevalencia de universitarios ansiosos que concluyen cursos de salud públicos y privados. Metodología: Se realizó un estudio transversal en Campina Grande-PB, de octubre 2013 a diciembre 2014, y tras la aprobación del Comité de Ética, se aplicó el Inventario de Ansiedad de Beck validado para el portugués y un cuestionario semi-estructurado. Los datos obtenidos fueron tratados en el Statistical Package for the Social Sciences en la versión 18, empleando un intervalo de confianza del $95 \%$ en los análisis calculados. Resultados: Fue encontrada una relación estadística significante entre los universitarios ansiosos y las variables horas de sueño por día, calidad del sueño, IES, insomnio, fatiga y miedo de quedarse solo. En el análisis de varianza, el curso de Farmacia obtuvo el mayor promedio en las puntuaciones del inventario. Conclusión: Se concluyó que la alta prevalencia de universitarios ansiosos indica la necesidad de políticas de atención a la salud mental de ese segmento poblacional, mejorando la calidad de vida en las universidades.

Palabras-clave: Salud mental. Estudiantes universitarios. Trastorno de ansiedad. 


\section{Introdução}

A ansiedade é um distúrbio comum que afeta o funcionamento emocional e psicossocial do indivíduo(1) podendo ser definida como uma experiência funcional persistente de interação com o meio ambiente(2) ou mesmo um estado adaptativo à situações de estresse, perigo real ou imaginário.(3) Dentre os sintomas de estados ansioso estão falta de ar, dores no peito, distúrbios do sono, fadiga, tensão muscular, dificuldade de concentração, palpitações, vertigens, náuseas, nervosismo e inquietação. A intensidade dos sintomas varia de acordo com a reatividade do indivíduo à situação ansiogênica. $(3,4)$

A morbidade relacionada a estados ansiosos vem aumentando expressivamente na população humana desde o último século. Esse fato tem associação com as profundas transformações ocorridas no âmbito econômico, social e cultural, sendo relacionada às situações vivenciadas pelos indivíduos em seu cotidiano. $(5,6)$

Ao ingressar na faculdade, os universitários são submetidos a uma grande carga de estresse, devido as longas horas de estudo, cobranças pessoais, de professores e familiares.(7)

Assim, estima-se que entre 14\% a $19 \%$ de universitários podem apresentar algum transtorno psicológico durante a vida acadêmica.(8) Tem-se, ainda, que em função de subdiagnósticos e até da baixa procura por tratamento, indivíduos com algum transtorno de ansiedade frequentemente perdem oportunidades de crescimento pessoal e profissional, desenvolvendo auto avaliação negativa e se isolando ainda mais da participação na sociedade.(9)

Dessa forma, o estudo teve como objetivo determinar a prevalência de alunos ansiosos dentre os concluintes de cursos da área de saúde ansiosos, de Instituições de Ensino Superior (IES) pública e privada, relacionando-os com os dados sociodemográficos e hábitos de vida.

\section{Métodos}

O estudo foi do tipo transversal, com abordagem quantitativa, realizado em duas IES, uma pública e outra privada, no Município de Campina Grande - PB, durante os meses de outubro de 2013 a dezembro de 2014. Participaram do estudo universitários concluintes dos cursos de educação física, farmácia e odontologia da IES pública e dos cursos de enfermagem, fisioterapia e nutrição da IES privada. Os participantes não foram desconsiderados ou discriminados por sexo, posição social, etnia ou religião.

Foram incluídos os alunos que estavam presentes em uma das duas tentativas de abordagem para participação do estudo, que possuíssem 18 anos ou mais, considerando-se as faltas após tentativas e recusas como perdas. Dos 155 matriculados e assíduos, obteve-se uma perda de $12,9 \%$ $(n=20)$, não havendo nenhuma por recusa. A participação no estudo foi iniciada após a apresentação da pesquisa e assinatura do Termo de Consentimento Livre e Esclarecido (TCLE).

Para determinação do perfil ansioso foi empregado o Inventário de Ansiedade de Beck ou Beck Anxiety Inventory $(B A I)$, na versão validada em português. O inventário é autoaplicável e apresenta 21 perguntas, sendo considerado um bom instrumento psicométrico para detecção de ansiedade, sem interferência de outros transtornos mentais menores, como a depressão. $(10,11)$

As perguntas contidas no inventário são de múltipla escolha, cada uma com um escore associado em uma escala: Nem um pouco (0), Ligeiramente (1), Moderadamente (2) e Severamente (3). A soma dos escores varia entre 0 e 63 pontos, sendo classificado nas faixas de perfil ansioso: 0 7 Mínimo; 8 - 15 Suave; 16-25 Moderado e 26-63 Grave.

Após o preenchimento do BAI, os participantes responderam a um questionário contendo informações acadêmicas (curso e turma), sociodemográficas (sexo, idade, cor da pele, estado civil, atividade profissional, local de residência e número de moradores do domicílio), condições de saúde (altura e peso para o cálculo do índice de massa corporal [IMC], horas de sono por dia, qualidade do sono e doenças referidas) e hábitos de vida (tabagismo, etilismo, atividade física, uso de substâncias ilícitas e horas de internet/televisão por dia).

Para o cálculo e classificação do IMC, a altura e o peso dos participantes foram autorreferidos e considerados os pontos de corte definidos pela Organização Mundial de Saúde (OMS): baixo peso $\left(<18,5 \mathrm{~kg} / \mathrm{m}^{2}\right)$, eutrofia $\left(18,5-24,9 \mathrm{~kg} / \mathrm{m}^{2}\right)$, sobrepeso $\left(25,0-29,9 \mathrm{~kg} / \mathrm{m}^{2}\right)$ e obesidade $\left(\geq 30 \mathrm{~kg} / \mathrm{m}^{2}\right)(12)$.

Para a variável horas de sono, o ponto de corte foi aquele no qual o estado ótimo de vigília é obtido, que corresponde a, em média, 7 horas de sono em um período de 24 horas(13). A qualidade do sono foi considerada uma variável subjetiva.

Em relação à atividade física foram considerados ativos os universitários que referiram no mínimo 30 minutos diários de atividade física moderada em cinco dias da semana nas horas de lazer ou 20 minutos diários em três dias da semana de atividade vigorosa, de acordo com o Questionário Internacional de Atividades Físicas (International Physical Activity Questionnaire - IPAQ).(14)

A classificação do consumo de bebidas alcoólicas seguiu o indicado pela OMS,(15) que especifica como sendo consumo leve o equivalente a 3 doses de destilados ou garrafas de fermentados; consumo moderado entre 3 e 7 doses e; consumo elevado acima de 7 doses. Considerando como doses/garrafas o equivalente a $350 \mathrm{~mL}$ de cerveja ou $150 \mathrm{~mL}$ de vinho ou $40 \mathrm{~mL}$ de destilados, consumidos na última semana. 
Foram considerados tabagistas todo indivíduo que, por ocasião do inquérito, estivesse fumando qualquer tipo ou quantidade de tabaco, diariamente há pelo menos seis meses.(16)

O tratamento estatístico foi realizado por meio do programa Statistical Package for The Social Sciences na versão 18.0 (SPSS 18). As variáveis quantitativas foram agrupadas em tabelas com frequências absolutas, frequências relativas e valores de $\mathrm{p}$ foram obtidos das análises bivariadas (Qui Quadrado e Regressão Linear Simples).

Para a comparação de médias entre grupos foi empregada a análise de variância (One Way ANOVA), obedecendo ao teste de homogeneidade de variância - Teste de Levene. $\mathrm{O}$ nível de significância para as correlações foi considerado $\mathrm{p}<0,05$ e intervalo de confiança de $95 \%$.

O estudo foi cadastrado na Plataforma Brasil e submetido à avaliação do Comitê de Ética em Pesquisa da Universidade Estadual da Paraíba, sendo aprovado sem pendências ou inadequações, CAAE parecer $n^{\circ} 16902013.5 .0000 .5187$.

\section{Resultados}

No total, participaram do estudo 135 universitários de cursos da área da saúde, com média de idade de 25,71 $( \pm 5,17)$ anos, estando matriculados $52,6 \%(n=71)$ em IES privada. Do total, $75,56 \%(\mathrm{n}=102)$ eram do sexo feminino, $79,3 \%(n=107)$ oriundos de cidades do Estado da Paraíba e os demais vindos de outros 13 estados brasileiros.

Na Figura 1 está representado o total de alunos matriculados por curso, estratificados por sexo.

Figura 1. Distribuição dos alunos por curso, estratificados por sexo.

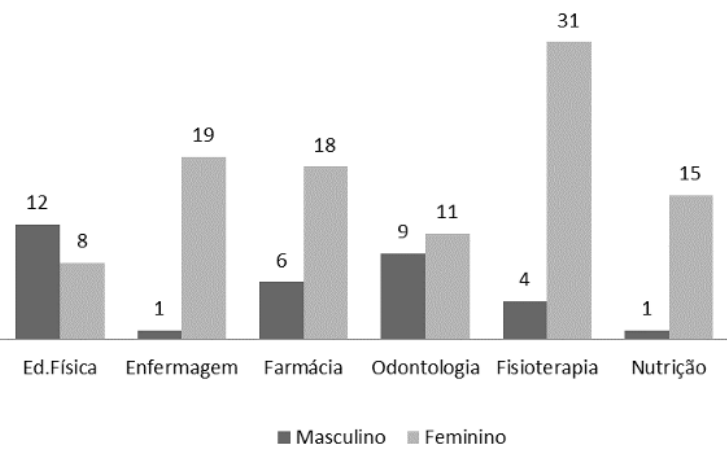

Fonte: Dados do estudo - Campina Grande, Paraíba, Brasil, 2014.

Em relação ao IMC, 9,6\% dos estudantes $(n=13)$ estavam com baixo peso, 69,6\% $(n=94)$ eutróficos, $18,5 \%$ $(n=25)$ com sobrepeso e 2,2\% $(n=3)$ com obesidade. Posteriormente, a classificação foi reagrupada em IMC normal (eutrófico) e IMC alterado (baixo peso, sobrepeso e obesidade), para as análises estatísticas analíticas.
Quando questionados se apresentavam alguma doença diagnosticada, 9,6\% $(\mathrm{n}=13)$ dos alunos declararam ao menos uma condição de saúde, sendo que desses $84,6 \%(n=11)$ eram do sexo feminino e dos diagnósticos: 59,9\% $(n=9)$ relataram doenças endócrinas, nutricionais e metabólicas, $33,3 \%(n=5)$ doenças do aparelho circulatório e 6,7\% $(n=1)$ doenças do aparelho respiratório.

A prevalência de indivíduos insuficientemente ativos foi maior que a de ativos, com 65,2\% $(n=88)$ dos estudantes declarando fazer menos de 30 minutos diários de atividade moderada nas horas de lazer, por cinco dias na semana.

Dos 39,3\% $(n=53)$ alunos que declararam consumir bebidas alcoólicas, $1,9 \%(\mathrm{n}=1)$ não consumiu bebidas na semana anterior ao dia de participação no estudo, 66,0\% $(n=35)$ tiveram um consumo leve de álcool, 20,8\% $(\mathrm{n}=11)$ consumo moderado e $11,3 \%(n=6)$ elevado.

Dos estudantes participantes, 3,7\% $(n=5)$ são fumantes e, quando questionados sobre uso de drogas ilícitas, 15,6\% $(n=21)$ declararam já ter consumido esse tipo de substância ao menos uma vez. Foram citadas como drogas consumidas loló/lança perfume em $60,0 \%$ das respostas $(n=15)$, maconha em 36,0\% (n=9) e ecstasy em 4,0\% (n=1).

\begin{tabular}{|c|c|c|c|c|c|c|}
\hline \multirow{2}{*}{ Variável } & \multicolumn{2}{|c|}{ Nấo Ansioso } & \multicolumn{2}{|c|}{ Ansioso } & \multirow[b]{2}{*}{ IC\% } & \multirow[b]{2}{*}{$\boldsymbol{p}$} \\
\hline & $\mathbf{N}$ & Fr\% & $\mathbf{N}$ & Fr\% & & \\
\hline \multicolumn{7}{|l|}{ IES } \\
\hline $\begin{array}{l}\text { Pública } \\
\text { Privada }\end{array}$ & $\begin{array}{l}28 \\
43\end{array}$ & \begin{tabular}{|l|}
43,8 \\
60,6
\end{tabular} & $\begin{array}{l}36 \\
28\end{array}$ & $\begin{array}{l}56,3 \\
39,4\end{array}$ & \multirow[t]{2}{*}{$0,994-3,920$} & 0,05 \\
\hline \multicolumn{6}{|l|}{ Sexo } & \\
\hline Masculino & 21 & 63,6 & 12 & 36,4 & \multirow{2}{*}{$0,143-1,204$} & \multirow{2}{*}{0,14} \\
\hline Feminino & 50 & 49,0 & 52 & 51,0 & & \\
\hline Cor & & & & & 1 & \multirow{3}{*}{0,39} \\
\hline Branca & 45 & 50,0 & 45 & 50,0 & \multirow{2}{*}{$0,665-2,816$} & \\
\hline $\begin{array}{c}\text { Não Branca } \\
\text { âncesidência }\end{array}$ & 26 & 57,8 & 19 & 42,2 & & \\
\hline \multicolumn{7}{|l|}{ Local de residência } \\
\hline Outra & $\begin{array}{l}50 \\
15\end{array}$ & $\begin{array}{l}50,9 \\
60,0\end{array}$ & $\begin{array}{l}34 \\
10\end{array}$ & $\begin{array}{l}49,1 \\
40,0\end{array}$ & \multirow[t]{2}{*}{$0,485-4,055$} & 0,41 \\
\hline \multicolumn{6}{|l|}{ Com quem mora } & \\
\hline Sozinho & 07 & 53,8 & 06 & 46,2 & \multirow{3}{*}{$0,113-2,055$} & \multirow{3}{*}{0,92} \\
\hline Acompanhado & 64 & 52,5 & 58 & 47,5 & & \\
\hline \multicolumn{5}{|l|}{$\begin{array}{c}\text { Atividade profissional } \\
\text { Estudante }\end{array}$} & & \\
\hline Estudante & 43 & 53,1 & 38 & 46,9 & \multirow{3}{*}{$0,228-1,489$} & \multirow{3}{*}{0,88} \\
\hline Estuda e Trabalha & 28 & 51,9 & 26 & 48,1 & & \\
\hline Estado civil & & & & & & \\
\hline Solteiro & 51 & 48,6 & 54 & 51,4 & $1,268-$ & \\
\hline Casado & 20 & 66,7 & 10 & 33,3 & 14,386 & 0,08 \\
\hline IMC & & & & & & \\
\hline Normal & 49 & 52,1 & 45 & 47,9 & & \\
\hline Alterado & 22 & 53,7 & 19 & 46,3 & $0,440-3, / 78$ & $0,8 /$ \\
\hline Horas de sono por dia & & & & & & \\
\hline Menos de 7 horas & 50 & 47,6 & 55 & 52,4 & & \\
\hline Mais de 7 horas & 21 & 70,0 & 09 & 30,0 & $0,163-0,927$ & 0,03 \\
\hline Qualidade do sono & & & & & & \\
\hline Satisfatória & 36 & 66,7 & 18 & 33,3 & & $<0,01$ \\
\hline Não satisfatória & 35 & 43,2 & 46 & 56,8 & $0,300-3,023$ & $\infty, 01$ \\
\hline Número de refeiçóes/dia & & & & & & \\
\hline Menos de 03 & 5 & 31,3 & 11 & 68,8 & $0,119-1,115$ & 0,06 \\
\hline 03 ou mais & 66 & 55,5 & 53 & 44,5 & $0,119-1,115$ & 0,00 \\
\hline Atividade Física & & & & & & \\
\hline Sedentário & 44 & 50,0 & 44 & 50,0 & & \\
\hline Ativo & 27 & 57,4 & 20 & 42,6 & $0,300-2,997$ & 0,40 \\
\hline $\begin{array}{l}\text { Horas de internet/tv por } \\
\text { dia }\end{array}$ & & & & & & \\
\hline Menos de 04 horas & 38 & 48,1 & 41 & 51,9 & $0,323-1,289$ & 0,21 \\
\hline $\begin{array}{l}\text { Mais de } 04 \text { horas } \\
\text { Ftilismo }\end{array}$ & 33 & 58,9 & 23 & 41,1 & & \\
\hline Etilismo & & & & & & \\
\hline Sim & 24 & 45,3 & 29 & 54,7 & & \\
\hline Nã̀o & 47 & 57,3 & 35 & 42,7 & $0,307-1,235$ & 0,17 \\
\hline Tabagismo & & & & & & \\
\hline Sim & 02 & 40,0 & 03 & 60,0 & $0,095-3,645$ & $0,66^{* * *}$ \\
\hline Não & 69 & 53,1 & 61 & 46,9 & $0,095-3,645$ & \\
\hline $\begin{array}{l}\text { Nota: Fonte: Dados do esth } \\
\text { IC\%=Intervalo de Confianc } \\
\text { Massa Corporal; *Qui Quad }\end{array}$ & to. Can & & & ra & sil, 2014. & dice de \\
\hline
\end{tabular}


Com a análise dos escores do $B A I$ observou-se que $18,5 \%(\mathrm{n}=25)$ dos universitários apresentavam perfil mínimo de ansiedade, 34,1\% $(\mathrm{n}=46)$ perfil suave, $34,1 \%$ $(n=46)$ moderado e $13,3 \%(n=18)$ grave. Para a estatística analítica, essa classificação foi dicotomizada em dois perfis: Não ansioso (mínimo e suave) e Ansioso (moderado e grave).

A Tabela 1 mostra as frequências absolutas e relativas das variáveis sociodemográficas e hábitos de vida dos 135 universitários, assim como o Intervalo de Confiança a 95\% e valores de $p$ para o Teste do Qui Quadrado de Pearson, ou empregando-se o Teste Exato de Fisher quando necessário.

Foi estatisticamente significante a relação entre o escore do $B A I$ e a variável horas de sono por dia, para os indivíduos que dormem menos de 07 horas, com o $\mathrm{p}=0,03$. Apesar da relação com as variáveis $I E S$ e qualidade do sono ter obtido um $\mathrm{p}<0,05$, não se configuram significantes para os intervalos de confiança considerados, já que não rejeitam a hipótese nula.

Também foram calculados os valores de p para o Qui Quadrado relacionando o escore do $B A I$ a outros fatores associados à ansiedade pela literatura, como sensação de confusão $(\mathrm{p}=0,07$ e $\mathrm{IC}=0,019-1,483)$, diminuição da coordenação motora $(\mathrm{p}=0,26 \mathrm{e} \mathrm{IC}=0,029-2,865)$, insônia $(\mathrm{p}<0,01$ e IC $=0,124-0,711)$, fadiga $(\mathrm{p}<0,01$ e $\mathrm{IC}=0,102-$ $0,451)$ e medo de ficar só ( $\mathrm{p}<0,01$ e IC $=0,01-0,710)$, sendo essas três últimas relações estatisticamente significantes.

Utilizando-se como ponto de corte os valores de $\mathrm{p}<0,20$, foram selecionadas as variáveis IES, sexo, estado civil, horas de sono por dia, qualidade do sono, número de refeições por dia, etilismo, insônia, fadiga e medo de ficar só para obtenção dos modelos de Regressão Linear Simples (RLS), expostos na Tabela 2.

Tabela 2: Regressão Linear Simples das correlações entre a pontuação do Escore do $B A I$ e variáveis independentes, considerando os 135 participantes.

\begin{tabular}{|c|c|c|c|c|c|c|}
\hline \multicolumn{2}{|r|}{ Variáveis } & $\mathbf{r}$ & $\mathbf{R}^{2}$ & $\mathbf{F}$ & $\begin{array}{l}\text { Durbin } \\
\text { Watso }\end{array}$ & $\boldsymbol{p}$ \\
\hline \multirow{10}{*}{$\begin{array}{c}\text { Classificaç } \\
\text { ão do } \\
\text { Escore do } \\
B A I^{*}\end{array}$} & IES & 0,168 & 0,028 & 3,869 & 1,838 & 0,05 \\
\hline & Sexo & 0,126 & 0,016 & 2,139 & 1,726 & 0,14 \\
\hline & Estado Civil & 0,151 & 0,023 & 3,089 & 1,791 & 0,08 \\
\hline & Horas de sono por dia & 0,186 & 0,035 & 4,784 & 1,850 & 0,03 \\
\hline & Qualidade do sono & 0,230 & 0,053 & 7,438 & 1,894 & $<0,01$ \\
\hline & $\begin{array}{c}\text { Número de refeições por } \\
\text { dia }\end{array}$ & 0,157 & 0,025 & 3,349 & 1,877 & 0,06 \\
\hline & Etilismo & 0,118 & 0,014 & 1,868 & 1,805 & 0,17 \\
\hline & Insônia & 0,242 & 0,058 & 8,263 & 1,832 & $<0,01$ \\
\hline & Fadiga & 0,360 & 0,130 & 19,811 & 2,020 & $<0,01$ \\
\hline & Medo de ficar só & 0,241 & 0,058 & 8,220 & 1,825 & $<0,01$ \\
\hline
\end{tabular}

Nota: Fonte: Dados do estudo. Campina Grande, Paraíba, Brasil, 2014

$B A I=B e c k$ AnxietyInventory; IES=Instituição de Ensino Superior.

Na RLS, observando o resultado do R2 (Coeficiente de Determinação), a variável preditora de maior interferência nos resultados do escore do BAI foi obtida no modelo correlacionando a fadiga $(13,0 \%)$, seguida por insônia e medo de ficar só (ambas 5,8\%) e qualidade do sono (5,3\%). O ajuste adequado das variáveis ao modelo de RLS foi verificado pelos valores de Durbin Watson próximos a 2, indicando não haver autocorrelação entre as variáveis. Apresentaram valores de p estatisticamente significantes as relações com
$I E S$, horas de sono por dia, qualidade do sono, insônia, fadiga e medo de ficar só.

Na Tabela 3 estão os resultados para a análise de variância simples (One Way ANOVA).

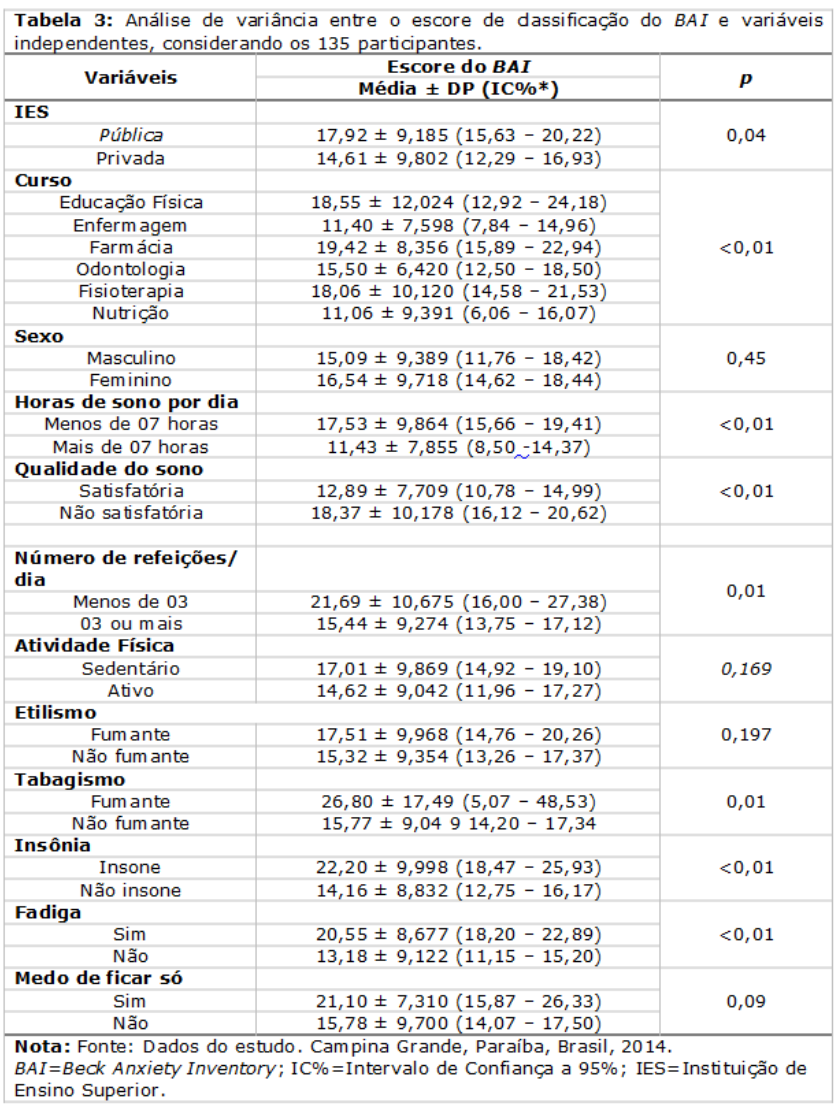

A média geral do escore do $B A I$ foi de $16,18 \pm 9,623$ pontos (IC 95\% = 14,54 - 17,82), resultando em um perfil geral de ansiedade moderada para a amostra. Universitários da IES pública, curso de farmácia, sexo feminino, qualidade do sono não satisfatória, consumir menos de três refeições por dia e ser fumante obtiveram as maiores médias de escore do BAI. Não houve significância estatística na comparação das médias apenas para as variáveis: sexo, atividade física, etilismo e medo de ficar só.

\section{Discussão}

Todo indivíduo apresenta um nível de ansiedade frente às situações de estresse ou adaptativas aos acontecimentos do cotidiano. O que define o comportamento como patológico é a intensidade e frequência que o organismo apresenta frente ao motivo que desencadeia o estado ansioso.(17)

Ao avaliar o resultado do escore do $B A I$, a população estudada apresenta uma prevalência de indivíduos ansiosos semelhante ao estudo de Prado, Kuberayashi e Silva(18) 
para universitários com perfil moderado de ansiedade, mas diferem quanto ao perfil grave, com percentual três vezes menor.

Após a recategorização do escore do $B A I$, aproximadamente metade de indivíduos que responderam ao inventário foram classificados no grupo com ansiedade, o que seria mais que o dobro da prevalência esperada de um transtorno psicológico para a população estudada.(9)

Deve-se levar em consideração que uma pontuação elevada por si só não determina que um indivíduo tenha um transtorno de ansiedade específico, mas é um forte preditor que indica a necessidade de uma avaliação mais detalhada. $(19,20)$

No estudo de Gama et al.(21) também houve correlação significativa entre o estado civil do indivíduo e a presença de ansiedade, sendo os solteiros mais propensos a perfis ansiosos.

As variáveis estado civil, número de refeições por dia, IMC, consumo de bebidas alcoólicas, tabagismo e prática de atividade física não apresentaram correlações de independência ou linearidade com o perfil ansioso. Entretanto, ao realizar uma análise individual dessas características, em estudo realizado por Carvalho e Martins et al.,(22) 18,2\% $(\mathrm{n}=110)$ dos universitários apresentavam sobrepeso ou obesidade e $52 \%(n=315)$ eram inativos ou insuficientemente ativos, percentuais em conformidade com este estudo.

Relatos na literatura indicam que a prática regular de atividade física é considerada um fator preventivo de estados ansiosos, por produzir efeitos antidepressivos e ansiolíticos, protegendo o organismo dos resultados prejudiciais do estresse na saúde física e mental.(23)

Para Araújo, Mello e Leite(24) apenas os exercícios que não ultrapassem o limite de força e resistência do indivíduo são adequados na melhoria e proteção contra alterações de humor e que os exercícios anaeróbios são considerados ansiogênicos.

Em relação ao uso de álcool, tabaco e outras drogas, em levantamento realizado junto a universitários de cursos de saúde de uma instituição particular foram verificados percentuais bem maiores de consumo dessas substâncias quando comparados a este estudo. No trabalho de Chiapetti $\mathrm{N}$, Serbena 70,0\% $(\mathrm{n}=376)$ dos participantes declararam consumir bebidas alcoólicas, 32,0\% ( $\mathrm{n}=172)$ serem tabagistas e 37,18\% ( $n=200)$ já haver feito uso de alguma substância ilícita, sendo maconha a droga mais relatada e dos motivos, o uso para diminuir a ansiedade e estresse foi o segundo mais prevalente.(25)

As variáveis relativas ao sono (horas de sono por dia, qualidade do sono e insônia) apresentaram correlações significantes seja na verificação da linearidade ou diferença de médias entre grupos, confirmando a forte relação desses fatores aos processos ansiosos. Poucas horas de sono por dia e sono de má qualidade podem levar, além de estados ansiosos, a cansaço, perda da concentração, fadiga, aumento da sensibilidade à dor, nervosismo, ideias irracionais, alucinações, perda de apetite, constipação e maior propensão a acidentes.(13)

\section{Conclusão}

O estudo reuniu informações relacionadas aos processos ansiosos de forma condensada e com resultados semelhantes a outros estudos envolvendo o mesmo segmento populacional, corroborando as associações encontradas junto aos grupos de maiores escores do BAI.

Não é de se estranhar que quase metade da amostra participante do estudo apresentou perfil moderado ou grave de ansiedade, descobertos mesmo com as dificuldades do estudo em encontrar tempo e espaço nas universidades adequado à aplicação dos instrumentos de coletas de dados e a disponibilidade de universitários concluintes presentes em um só ambiente.

Verifica-se a necessidade de outras pesquisas, pelas quais sejam feitas avaliações psicológicas para confirmação dos resultados e diagnósticos diferenciais, classificando os possíveis transtornos de ansiedade apresentados.

É importante que as políticas de saúde mental não estejam restritas aos centros de atenção psicossocial para os indivíduos com transtornos mentais maiores.

Políticas de saúde que sejam direcionadas a grupos como o segmento populacional abordado, com intuito preventivo do desenvolvimento de condições mais graves que os estados ansiosos, são necessárias ajudando na formação de profissionais qualificados, mais seguros e confiantes e, assim, melhorando a qualidade de vida dentro das universidades.

\section{Referências}

1. Balestrieri M, Isola M, Quartaroli M, Roncolato M, Bellantuono C. Avaliando transtorno de ansiedadedepressiva mista. Uma pesquisa nacional de cuidados primários. Psychiatry Research. 2010;176:197-201.

2. Antonio WZ. Características básicas do transtorno de ansiedade generalizada. Medicina (Ribeirão Preto). 2017;50(Supl. 1):51-5.

3. Cabral R, Nardi AE. Anxiety and inhibition of panic attacks within translational and prospective research contexts. Trends Psychiatry Psychother. 2012;34(2):62-72.

4. Sales LF, Silva MJP. Efeito das essências florais em indivíduos ansiosos. ACTA Paul Enferm. 2012;25(2):23842.

5. Hernandez JAE, Santos GR, Silva JO, Mendes SLL, Ramos VCB. Evidências de Validade da Escala de Ansiedade em Estatística em Alunos da Psicologia. Psicol. cienc. prof. 2015; 35(3):659-75. 
6. José LHA, Vivian AG, José FEM, Souza, FP. Ansiedade, estresse, sintomas de TDAH e desempenho em candidatos no exame da Ordem dos Advogados do Brasil/RS. Aletheia. 2015;47:142-54.

7. Sgaribold AR, Puggina ACG, Silva MJP. Análise da percepção dos professores em relação aos sentimentos dos alunos em sala de aula. Rev Esc Enferm USP. 2011;45(5):1206-12.

8. Cerchiari EAN, Caetano D, Faccenda O. Prevalência de transtornos mentais menores em universitários. Estud Psicol. 2005;10(3):413-20.

9. Levitan MN, Chagas MHN, Crippa JAS, Manfro GG, Hetem LAB, Andrada MC et al. Diretrizes da Sociedade Médica Brasileira para o tratamento do transtorno de ansiedade social. Rev Bras Psiquiatr. 2011;33(3):292-302.

10. Beck AT, Epstein N, Brown G, Steer RA. An inventory for measuring clinical anxiety: Psychometric properties. J Consult Clin Psychol. 1988;56(6):893-97.

11. Quintão S, Delgado AR, Pietro G. Validity study of the Beck Anxiety Inventory (Portuguese version) by the Rash Rating Scale Model. Psicol Reflex Crit. 2013;26(2):305-10.

12. World Health Organization. Obesity: preventing and managing the global epidemic. Report of a World Health Organization Consultation. Geneva (SWZ): WHO, 2004.

13. Costa SV, Ceolim MF. Fatores que interferem na qualidade do sono de pacientes internados. Rev Esc Enferm USP. 2013;47(1):46-52.

14. Sousa CA, César, CLG, Barros MBA, Carandina L, Goldbaum M, Marchioni DML, Fisberg RM. Prevalência de atividade física no lazer e fatores associados: estudo de base populacional em São Paulo, Brasil, 2008-2009. Cad. Saúde Pública. 2013;29(2):270-82.

15. World Health Organization. Global status report on alcohol. Report of a World Health Organization Consultation. Geneva (SWZ): WHO, 2011.
16. Freitas ERFS, Araújo ECLS, Alves KS. Influência do tabagismo na força muscular respiratória em idosos. Fioster Pesqui. 2012;19(4):326-31.

17. Braga JEF, Pordeus LC, Silva ATMC, Pimenta FCF, Diniz MFFM, Almeida RN. Ansiedade patológica: bases neurais e avanços na abordagem psicofarmacológica. $\mathrm{R}$ Bras Ci Saúde. 2010;14(2):93-100.

18. Prado JM, Kurebayashi LFS, Silva MJP. Eficácia da auriculoterapia na redução de ansiedade em universitários de enfermagem. Rev Esc Enferm. USP. 2012;48(6):120008 .

19. Leyfer OT, Ruberg JL, Woodruff-Borden J. Examination of the validity of the Beck anxiety inventory and its factors as a screener for anxiety disorders. J Anxiety Disord. 2006;20:444-58.

20. Pigott TA. Anxiety disorders in women. Psychiatr Clin North Am. 2003;26(3):621-72.

21. Gama MMA, Moura GS, Araújo RF, TeixeiraSilva F. Ansiedade-traço em universitários universitários de Aracajú (SE). Rev Psiquiatr Rio Gd Sul. 2008;30(1):19-24.

22. Carvalho e Martins MC, Ricarte IF, Rocha CHL, Maia RB, Silva VB, Veras AB, et al. Pressão arterial, excesso de peso e nível de atividade física em universitários de universidade pública. Arq. Bras. Cardiol. 2010;95(2):19299.

23. Salmon P. Effects of physical exercise on anxiety, depression, and sensitivity to stress: A unifying theory. Clinl Psychol Rev. 2001;21(1):33-61.

24. Araújo SRC, Mello MT, Leite JR. Transtornos de ansiedade e exercício físico. Rev Bras Psiquiatr. 2007;29(2):164-71.

25. Chiapetti N, Serbena CA. Uso de álcool, tabaco e drogas por universitários da área de saúde de uma universidade de Curitiba. Psicol Reflex Crit. 2007;20(2):303-13. 